\title{
Relationship between resistin and IL-23 levels in follicular fluid in infertile patients with endometriosis undergoing IVF-ET
}

\author{
Qun-Fang Zhang ${ }^{1, A, C, D}$, Guo-Yong Chen 2, B, Yun Liu ${ }^{2, B}$, Hui-Juan Huang 2, B, Yan-Feng Song ${ }^{2, ~ B ~}$ \\ ${ }^{1}$ Department of Obstetrics and Gynecology, Fuzhou General Hospital of Nanjing Military Command, China \\ ${ }^{2}$ Reproductive Medicine Center, Department of Obstetrics and Gynecology, Fuzhou General Hospital of Nanjing Military Command, China \\ A - research concept and design; B - collection and/or assembly of data; C - data analysis and interpretation; \\ $D$ - writing the article; $E$ - critical revision of the article; $F$ - final approval of the article
}

\section{Address for correspondence \\ Qun-Fang Zhang}

E-mail: qunfangzhang111@126.com

\section{Funding sources}

Fund of Medical Science and Technology

of Nanjing Military Command, Fuzhou, China

(2013-MS125). Fund of Science and Technology

of Fuzhou General Hospital, China (201212).

Conflict of interest

None declared

Received on March 26, 2015

Reviewed on May 7, 2015

Accepted on June 3, 2015

DOI

10.17219/acem/41149

\section{Copyright}

Copyright by Author(s)

This is an article distributed under the terms of the

Creative Commons Attribution Non-Commercial License

(http://creativecommons.org/licenses/by-nc-nd/4.0/)

\section{Abstract}

Background. Endometriosis (EM) interferes with the reproductive process and affects the success rate of in vitro fertilization (IVF). Inflammatory cytokines are suggested to play a role in infertility in patients with EM.

Objectives. In this study, we aimed to investigate the relationship between resistin and interleukin 23 (IL-23) levels in follicular fluid (FF) and serum together with the severity of endometriosis and in vitro fertilization/embryo transfer (IVF-ET) outcome.

Material and methods. Samples from 116 infertile women were studied using enzyme-linked immunosorbent assay (ELISA). The study group consisted of 76 infertile patients diagnosed with EM (40 with stages $|-| \mid$ and 36 with stages III-IV) undergoing IVF-ET. The control group included 40 women with tubal factor infertility. FF and serum samples were collected on the day of follicle aspiration and hCG administration, respectively.

Results. The serum and FF resistin levels were significantly higher in the EM group than in the control group ( $p$-value $<0.05)$. The FF resistin and IL-23 levels were significantly higher in EM stages III-IV than in stages I- II ( $p$-value $<0.05)$, and the serum resistin and IL-23 levels were also significantly ( $p$-value $<0.01$ ) higher in stages III-IV than in stages $\mathrm{I}-\|$. The $\mathrm{E}_{2}$ level on the day of $\mathrm{hCG}$ administration and the implantation rate were both significantly lower in the EM group than in the control group. However, there were no differences in the Gn duration and dose, and the cleavage, implantation and clinical pregnancy rates between the 2 groups.

Conclusions. Our results suggest that patients with EM exhibit increased resistin level in FF and serum. Advanced EM may contribute to infertility via decreased embryo implantation rates because of inflammation and immune rejection. No influence was observed on pregnancy outcomes after IVF-ET.

Key words: endometriosis, IVF-ET, resistin, IL-23, pregnancy outcome 


\section{Introduction}

Inflammatory cytokines are suggested to play a role in infertility in patients with endometriosis (EM). Previously, a meta-analysis carried out on the effects of endometriosis on in vitro fertilization/embryo transfer (IVF/ET) outcomes concluded that EM interferes with all aspects of the reproductive process, and the success rate of in vitro fertilization (IVF) in EM patients was almost half compared to women without EM. ${ }^{1}$ However, the mechanism by which EM affects fertility in IVF remains unclear. Human follicular fluid forms the microenvironment of the developing oocyte and has an important influence on oocyte quality, embryo development, and implantation. ${ }^{2}$ In this study, we measured the follicular fluid (FF) and serum concentrations of resistin and interleukin 23 (IL-23) in EM and control patients and in different stages of EM, and retrospectively assessed IVF-ET outcomes in order to understand the possible impact of EM on IVF-ET outcomes.

\section{Material and methods}

\section{Patients}

This study was approved by the Ethics Committee on Human-Assisted Reproduction of Fuzhou General Hospital, and informed consent was obtained from all the study participants.

This was a randomized controlled retrospective database-search study of a total of 116 infertile patients from January 2012 to December 2012. The patients were 22 to 38 years old (mean age $30.67 \pm 4.32$ ). The study group consisted of 76 women diagnosed with EM (40 with stages I-II and 36 with stages III-IV) with no other factor than EM causing infertility. The control group consisted of 40 women with tubal factor infertility. A total of 25 , 15,19 , and 17 women in the study group had stages I, II, III, and IV EM, respectively. The EM was confirmed by laparoscopy, and all the patients were treated surgically. The patients in the EM group were scored according to the revised American Fertility Society (AFS) classification (1997). ${ }^{3}$ Based on the observation of ectopic endometrial parts in laparoscopic surgery, the number, size, degree of adhesion rate, and scores of stages I-IV EM patients were $1-5,6-15,16-40$, and $>40$, respectively. Clinical and laboratory data was analyzed.

\section{Inclusion criteria}

Patients were recruited to the study based on the following inclusion criteria: (1) women under the age of 38 years; (2) those with $\geq 10$ punctured follicles or retrieved oocytes; (3) those who were receiving IVF-ET for the first time; (4) underwent transfer of 1-2 embryos; and (5) had not received drugs affecting the function of the hypothalamus-pituitary-ovarian axis for at least 3 months before the study.

\section{Exclusion criteria}

The following exclusion criteria were applied: (1) women above the age of 38 years; (2) those with basic FSH (bovine follicle-stimulating hormone, bFSH) levels $>11.0 \mathrm{mIU} / \mathrm{m}$; (3) patients with autoimmune disease, liver disease, diabetes, or malignant tumors; (4) reproductive organ dysplasia; (5) acute pelvic inflammation and other changes confirmed by laparoscopy; and (6) inadequate data for analysis.

\section{Controlled ovarian stimulation}

Pituitary-ovarian suppression was achieved by administration of the standard "long protocol" gonadotropinreleasing hormone analogue $(\mathrm{GnRH})$ agonist with triptorelin acetate (Decapeptyl, Ferring, St. Prex, Switzerland) starting in the midluteal phase of the preceding cycle. When complete pituitary desensitization was confirmed by low plasma $E_{2}$ levels $\leq 30 \mathrm{pg} / \mathrm{mL}$, LH level $\leq 2 \mathrm{mIU} / \mathrm{mL}$, $\mathrm{E} 2<30 \mathrm{pg} / \mathrm{mL}$ and endometrial thickness $<5 \mathrm{~mm}$, the dosage was decided according to the age, basal endocrine, anti-Müllerian hormone (AMH), antral follicle count (AFC), and body mass index (BMI).

Ovarian stimulation was initiated by administration of recombinant FSH (Gonal-F, Serono, Switzerland; or Puregon, Schering-Plough, USA). The stimulated cycles were monitored by daily transvaginal ultrasonography (TVS) and intermittent assessment of the serum $\mathrm{E}_{2}$ levels. The daily gonadotrophin dose was customized based on an individual dose-response scheme. Final maturation of oocytes was induced by injection of $250 \mu \mathrm{g}$ of recombinant human chorionic gonadotrophin (hCG; Ovidrel, Serono, Switzerland) when the serum $E_{2}$ level was $\geq 150 \mathrm{pg} /$ dominant follicle and the 2 leading follicles reached a mean diameter of $18 \mathrm{~mm}$. The oocytes were retrieved transvaginally $34-36 \mathrm{~h}$ after hCG administration. Usually less than 2 best-quality embryos were transferred on day 3 after oocyte retrieval, and any extra good-quality embryos were cryopreserved for subsequent frozen-embryo transfer (FET) cycles.

\section{Collection of serum and follicular fluid}

Serum samples were collected on the day of hCG trigger. Ovarian follicular fluid samples were collected during oocyte retrieval by follicle aspiration using transvaginal puncture assisted by ultrasonic guidance. All visible follicles were aspirated separately. Samples of yellow, clear FF were centrifuged at $1500 \mathrm{rpm}$ for $10 \mathrm{~min}$. The clear supernatants were stored immediately at $-80^{\circ} \mathrm{C}$. Only material from the first 2 or 3 aspirated follicles was used in order to avoid contamination with blood. No contaminated samples were used for further analysis. 


\section{Quantification of resistin and IL-23}

Resistin and IL-23 ELISA kits were purchased from Xin Bo Sheng Biotechnology Co. The operation was carried out according to the kit instructions.

\section{High-quality embryos and pregnancy outcome}

Good-quality embryos were identified as 2 pn, with at least 7 blastomeres and $\leq 20 \%$ fragmentation. One or 2 embryos were chosen for transfer to the uterus under vaginal ultrasound guidance. The serum hCG was measured 2 weeks after embryo transfer to diagnose pregnancy, and the pregnancy was clinically confirmed by ultrasound at the $4^{\text {th }}$ week after ET. Transplantation was canceled in the case of uterine cavity effusion, endometrial thickness $<7 \mathrm{~mm}, \mathrm{E}_{2}$ on hCG day $>5000 \mathrm{pmol} / \mathrm{L}$, and/or oocyte number $>15$.

Table 1. Comparison of follicular fluid and serum resistin and IL-23 levels between patients in the endometriosis and control groups

\begin{tabular}{l|c|c|c|c|c|}
\multirow{2}{*}{ Indication } & \multirow{2}{*}{ Cases } & \multicolumn{2}{|c|}{ Resistin $(\mathrm{pg} / \mathrm{mL})$} & \multicolumn{2}{c}{ IL-23 $(\mathrm{pg} / \mathrm{mL})$} \\
\cline { 3 - 6 } & & follicular fluid & serum & follicular fluid & serum \\
\hline EM & 76 & $140.66 \pm 16.14$ & $33.44 \pm 28.26$ & $18.03 \pm 11.68$ & $18.37 \pm 12.16$ \\
\hline Control & 40 & $42.66 \pm 12.32$ & $13.20 \pm 6.09$ & $16.38 \pm 2.17$ & $16.38 \pm 2.17$ \\
\hline t & - & 2.58 & 2.462 & 0.487 & 0.564 \\
\hline P & - & 0.012 & 0.016 & 0.628 & 0.574 \\
\hline
\end{tabular}

Table 2. Comparison of follicular fluid and serum resistin and IL-23 levels of patients with different stages of EM (I-II and III-IV)

\begin{tabular}{l|c|c|c|c|c|}
\multirow{2}{*}{ Indication } & \multirow{2}{*}{ Cases } & \multicolumn{2}{|c|}{ Resistin $(\mathrm{pg} / \mathrm{mL})$} & \multicolumn{2}{c}{ IL-23 $(\mathrm{pg} / \mathrm{mL})$} \\
\cline { 3 - 6 } & & follicular fluid & serum & follicular fluid & serum \\
\hline I, II & 40 & $54.64 \pm 8.64$ & $14.04 \pm 6.21$ & $13.56 \pm 1.11$ & $13.48 \pm 1.08$ \\
\hline III, IV & 36 & $130.88 \pm 21.81$ & $52.86 \pm 28.40$ & $22.99 \pm 15.58$ & $23.26 \pm 15.80$ \\
\hline t & - & 8.909 & -8.231 & 3.822 & -3.806 \\
\hline P & - & 0.012 & 0.000 & 0.000 & 0.000 \\
\hline
\end{tabular}

Table 3. General data and clinical control ovarian stimulation outcomes of the patients in the 2 groups $(\bar{x} \pm s)$

\begin{tabular}{|l|c|c|c|}
\multicolumn{1}{|c|}{ Indicators } & $\mathrm{EM}(\mathrm{n}=76)$ & Control $(\mathrm{n}=40)$ & p-value \\
\hline Age (years) & $31.24 \pm 4.46$ & $30.37 \pm 3.53$ & 0.218 \\
\hline Infertility duration & $5.02 \pm 3.42$ & $4.89 \pm 2.60$ & 0.769 \\
\hline Body mass index & $22.34 \pm 3.26$ & $23.35 \pm 4.18$ & 0.376 \\
\hline Down-regulation duration (d) & $18.12 \pm 4.26$ & $19.4 \pm 6.22$ & 0.258 \\
\hline Gn duration (d) & $10.82 \pm 1.33$ & $11.51 \pm 1.08$ & 0.130 \\
\hline $\begin{array}{l}\text { Gn dosage (Amp) } \\
\text { hCG injection day } \\
\text { endometrial thickness (mm) } \\
\text { E2 (pg/mL) }\end{array}$ & $33.88 \pm 8.34$ & $35.12 \pm 11.02$ & 0.404 \\
\hline
\end{tabular}

\section{Statistical analysis}

All results are expressed as mean \pm SE. All data was analyzed using Statistical Package for Social Sciences (SPSS) v. 16.0. Student's t-test (for 2 groups) and analysis of variance (ANOVA; for more than 2 groups) were used for statistical analysis. The clinical pregnancy rates of the different groups were analyzed using the Student's t-test. P-values of $<0.05$ were considered significant.

\section{Results}

\section{Resistin and IL-23 levels in FF and serum in the 2 groups}

Table 1 presents the FF and serum resistin and IL-23 levels in both groups. The resistin levels in the FF and serum were significantly higher in the EM group than in the control group $(\mathrm{p}<0.05)$. The FF and serum IL-23 levels showed an increasing trend in the EM group compared to the control group, but this difference was not statistically significant $(\mathrm{p}>0.05)$.

\section{FF and serum resistin and IL-23 levels in different stages of EM}

Table 2 presents the FF and serum resistin and IL-23 levels in different stages of EM (groups I-II and III-IV). The resistin and IL-23 levels in the FF was significantly higher in EM stages III-IV than in EM stages I-II $(\mathrm{p}<0.05)$. The resistin and IL-23 levels in the serum were also significantly higher in EM stages III-IV than in stages I-II ( $\mathrm{p}<0.01)$.

\section{General information}

Tables 3 and 4 present the general data and clinical COS outcomes of the patients in the 2 groups. The $\mathrm{E}_{2}$ level on the day of hCG administration and the implantation rate were significantly lower in the EM group than in the control group $(\mathrm{p}<0.05)$. However, the downregulation duration, Gn dose, Gn duration, rate of fertilization, cleavage and clinical pregnancy, ovarian hyperstimulation syndrome (OHSS) and abortion showed no statistical significance between the 2 groups. 


\section{Discussion}

Endometriosis is a chronic disease characterized by the presence of endometrium-like tissue outside the uterus, most commonly on the ovary. It affects $10-15 \%$ of women of reproductive age. ${ }^{4}$ Previous studies have reported that $30-40 \%$ of infertile women have endometriosis, and these women are 20 times more likely to have EM than fertile women. ${ }^{5,6}$

EM is a complex genetic diseases whose pathogenesis is closely related to heredity, immunity, hormones, and environment. ${ }^{7,8}$ The mechanism by which EM causes infertility is yet to be understood, and it may be due to the ectopic lesions caused by EM on oocytes and embryos and other harmful endometrial factors that may affect the quality of embryos and implantation. There is increasing evidence suggesting that grow rate of embryos drop in patients with EM. ${ }^{8}$

Resistin is known as a fat tissue-specific factor and is closely related to the inflammatory response. Yi et al. determined that the mean concentration of PF resistin was significantly higher in women with endometriosis compared to the controls. ${ }^{9}$ The resistin levels increase during acute inflammation and have been found to be positively associated with inflammatory factors such as C-reactive protein (CRP). ${ }^{10,11} \mathrm{~A}$ meta-analysis of the resistin levels in 6636 patients reported that the resistin levels were higher in women than in men. ${ }^{12}$

IL-23 is a type of inflammatory cytokine that is known to participate in autoimmune diseases by promoting inflammation. ${ }^{13,14}$ Ours is the first study to explore the mechanism of EM fertility by determining the relationship between FF and serum resistin and IL-23 expression levels and the IVF/ET pregnancy outcome in EM patients.

The FF and serum resistin levels were significantly higher in the EM group than in the control group $(\mathrm{p}<0.05)$. The FF and serum IL-23 levels in the EM group

Table 4. Comparison of IVF/ET outcomes between patients in the 2 groups $(\bar{x} \pm s)$

\begin{tabular}{|l|c|c|c|}
\multicolumn{1}{|c|}{ Indicators } & EM $(\boldsymbol{n}=76)$ & Control $(\boldsymbol{n}=40)$ & $p$-value \\
\hline Fertilization rate (\%) & $88.76 \pm 22.00$ & $88.05 \pm 18.9$ & 0.856 \\
\hline Cleavage rate (\%) & $98.86 \pm 12.33$ & $97.28 \pm 6.08$ & 0.286 \\
\hline Good-quality embryos rate (\%) & $70.86 \pm 38.11$ & $72.72 \pm 24.30$ & 0.703 \\
\hline No. of embryos transferred & $1.99 \pm 0.22$ & $1.99 \pm 0.11$ & 0.250 \\
\hline Implantation rate (\%) & $18.33(22 / 120)$ & $31.43(22 / 70)$ & $p<0.05^{\mathrm{a}}$ \\
\hline Clinical pregnancy rate (\%) & $33.87 \%(21 / 62$ & $52.78(19 / 36)$ & $p>0.05^{\mathrm{b}}$ \\
\hline OHSS rate (\%) & $1.61(1 / 62)$ & $0(0 / 36)$ & 0.63 \\
\hline Abortion rate (\%) & $19.04 \%(4 / 21)$ & $10.53(2 / 19)$ & $p<0.05^{\mathrm{c}}$ \\
\hline
\end{tabular}

${ }^{a} x^{2}=4.26{ }^{b} x^{2}=3.37 ;^{c} x^{2}=0.57$ showed an increasing trend compared to the control group, although this difference was not significant ( $\mathrm{p}>0.05$ ).

Chen et al. studied the resistin levels in patients with and without and they suggested that the serum resistin levels might be a good predictor of ovarian response in infertile women without polycystic ovary syndrome (PCOS) during IVF. ${ }^{15}$ Further studies should investigate the role of serum resistin in response to inflammation caused by EM or chronic pelvic infection, both of which are major causes of female infertility. The findings of this study suggest a potent role of resistin in endometriosis. Further studies are needed to elucidate the biological implications of resistin in EM. The role of serum resistin in response to the inflammation caused by endometriosis or chronic pelvic infection should be examined in closer detail.

We also determined that EM was associated with increased levels of follicular IL-23. This result was not surprising. Impaired follicular fluid microenvironment characterized by elevated inflammatory cytokines may be the cause for poor oocyte quality, which could lead to poor IVF outcomes in patients with endometriosis. ${ }^{14,15}$

The FF resistin and IL-23 levels were significantly higher $(\mathrm{p}<0.05)$ in patients with severe EM (stages III and IV) than in patients with milder EM (stages I-II). Similarly, the serum resistin and IL-23 levels were significantly higher $(\mathrm{p}<0.01)$ in patients with severe EM (stages III and IV) than in patients with milder EM (stages I-II). Andreoli et al. measured the IL-23 levels in the serum and peritoneal fluid of women with minimal or mild EM and compared them with the corresponding levels in control subjects without endometriosis. ${ }^{16}$ Higher IL-23 levels were encountered in the peritoneal fluid of women with EM, suggesting a possible role of this cytokine in infertility in these women.

Many retrospective studies have investigated the IVF/ET outcomes in women with EM.117-19 Harkki et al. described that the $G$ n duration and $G$ n dose increased while the $E_{2}$ level decreased in patients with EM. ${ }^{17}$ We observed similar results in the $\mathrm{E}_{2}$ level in EM patients on the day of hCG administration $(2239.68 \pm 788.9 \mathrm{pg} / \mathrm{mL}$ vs $2556.80 \pm 1050.76 \mathrm{pg} / \mathrm{mL}$; $\mathrm{p}>0.05$ ), which was lower than the corresponding level in the control group. Kuivasaari et al. reported that the oocyte recovery, implantation, and clinical pregnancy rates were significantly lower in EM patients than in control subjects, which was similar to our findings: we observed a lower implantation rate in EM patients. ${ }^{18}$

In a meta-analysis, Barnhart et al. found that the implantation and clinical pregnancy rates were lower in the EM group than in the control group. ${ }^{1}$ Similar to these findings, we found that 
the implantation rate in the EM group (18.33\%, 22/120) was significantly lower $(\mathrm{p}<0.05)$ than that in the control group $(31.43 \%, 22 / 70)$, suggesting that the higher resistin and IL-23 levels may cause embryo implantation failure. Pouly et al. found that the number of recovered oocytes was lower in the group of patients with EM than in the control group. ${ }^{19}$

Neeta et al. compared the ovarian stimulation characteristics between women with EM and tubal infertility. ${ }^{20}$ The number of oocytes retrieved and the fertilization rate were significantly lower in the EM group than in the tubal group, but there was no significant difference in the percentage of metaphase II (M2) oocytes, cleavage rate, and percentage of grade 1 embryos formed between the 2 groups. Moreover, the mean number of embryos transferred did not differ between the 2 groups. The clinical pregnancy rate between the 2 groups was comparable.

The duration of GnRH-a downregulation, Gn dose, Gn duration, rate of fertilization, rate of cleavage, clinical pregnancy rate, OHSS, and abortion rate between the EM group and control group during IVF/ET therapy were compared, and the differences were not statistically significant. The clinical pregnancy rate in the EM and control groups were $33.87 \%$ (21/62) and 52.78\% (19/36), respectively, and although this difference was not of statistical significance $(\mathrm{p}>0.05)$, the EM group had a lower implantation rate.

Finally, EM is the cause of infertility where multiple factors are involved, and the high expression levels of resistin and IL-23 lead to inflammation and play an important role in the formation of EM; thus, inhibition of inflammation and improving the IVF-ET implantation rate and clinical pregnancy rate may be effective methods in treating EM.

\section{References}

1. Barnhart K, Dunsmoor-Su R, Coutifaris C. Effect of endometriosis on in vitro fertilization. Fertil Steril. 2002;77(6):1148-1155.

2. Tamura H, Takasaki A, Miwa I, et al. Oxidative stress impairs oocyte quality and melatonin protects oocytes from free radical damage and improves fertilization rate. J Pineal Res. 2008;44(3):280-287.

3. Damario MA, Rock JA. Classification of endometriosis. Semin Reprod Endocrinol. 1997;15(3):235-244.

4. Ozkan S, W. Murk W, Arici A. Endometriosis and infertility: Epidemiology and evidence-based treatments. Ann N Y Acad Sci. 2008;1127:92-100.

5. Kennedy S, Bergqvist A, Chapron C, et al. ESHRE guideline for the diagnosis and treatment of endometriosis. Hum Reprod. 2005;20(10):2698-2704.

6. Opøien HK, Fedorcsak P, Omland AK, et al. In vitro fertilization is a successful treatment in endometriosis-associated infertility. Fertil Steril. 2012;97(4):912-918.

7. Cho S, Choi YS, Jeon YE, et al. Expression of vascular endothelial growth factor (VEGF) and its soluble receptor-1 in endometriosis. Microvasc Res. 2012;83(2):237-242.

8. Bellelis P, Podgaec S, Abrao MS. Environmental factors and endometriosis. Rev Assoc Med Bras. 2011;57(4):448-452.

9. Yi KW, Shin JH, Park HT, et al. Resistin concentration is increased in the peritoneal fluid of women with endometriosis. Am J Reprod Immunol. 2010;64(5):318-323.
10. Cho $\mathrm{Y}$, Lee $\mathrm{SE}$, Lee $\mathrm{HC}$, et al. Adipokine resistin is a key player to modulate monocytes, endothelial cells, and smooth muscle cells, leading to progression of atherosclerosis in rabbit carotid artery. J Am Coll Cardiol. 2011;57(1):99-109.

11. Fargnoli JL, Sun Q, Olenczuk D, et al. Resistin is associated with biomarkers of inflammation while total and high-molecular weight adiponectin are associated with biomarkers of inflammation, insulin resistance, and endothelial function. Eur J Endocrinol. 2010;162(2):281-288.

12. Cabrera de Leon A, Almeida González D, González Hernández A, et al. The association of resistin with coronary disease in the general population. J Atheroscler Thromb. 2014;21(3):273-281.

13. Iwakura $\mathrm{Y}$, Ishigame $\mathrm{H}$. The IL-23/LL-17 axis in inflammation. $J$ Clin Invest. 2006;116(5):1218-1222.

14. Xu H, Schultze-Mosgau A, Agic Admir, et al. Regulated upon activation, normal T cell expressed and secreted (RANTES) and monocyte chemotactic protein 1 in follicular fluid accumulate differentially in patients with and without endometriosis undergoing in vitro fertilization. Fertil Steril. 2006;86(6):1616-1620.

15. Chen YC, Tsai EM, Chen HS, et al. Serum resistin level is a predictor of ovarian response in in vitro fertilisation cycle. Acta Obstet Gynecol Scand. 2007;86(8):963-967.

16. Andreoli CG, Genro VK, Souza CA, et al. T helper (Th)1, Th2, and Th17 interleukin pathways in infertile patients with minimal/mild endometriosis. Fertil Steril. 2011;95(8):2477-2480.

17. Harkki $P$, Tiitinen A, Ylikorkala O. Endometriosis and assisted reproduction techniques. Ann N Y Acad Sci. 2010;1205:207-213.

18. Kuivasaari $P$, Hippelainen $M$, Anttila $M$, et al. Effect of endometriosis on IVF/ICSI outcome: Stage III/IV endometriosis worsens cumulative pregnancy and live-born rates. Hum Reprod. 2005;20(11):3130-3135.

19. Pouly JL, Canis $M$, Velemir $L$, et al. Endometriosis-related infertility. $J$ Gynecol Obstet Biol Reprod (Paris). 2007;36(2):151-161.

20. Singh N, Lata K, Naha M, et al. Effect of endometriosis on implantation rates when compared to tubal factor in fresh non donor in vitro fertilization cycles. J Hum Reprod Sci. 2014;7(2):143-147. 